\title{
Space-Frequency Block Code for MIMO-OFDM Communication Systems with Reconfigurable Antennas
}

\author{
Vida Vakilian*, Jean-François Frigon*, and Sébastien Roy ${ }^{\dagger}$ \\ * École Polytechnique de Montréal, Dept. of Electrical Engineering, Montréal, QC, H3T 1J4, Canada \\ \{vida.vakilian, j-f.frigon\}@ polymtl.ca \\ $\dagger$ Dept. of Elec. and Comp. Engineering, Université Sherbrooke Sherbrooke, QC, Canada \\ sebastien.roy13@usherbrooke.ca
}

\begin{abstract}
We propose a space-frequency (SF) block coding scheme for a multiple-input multiple-output (MIMO) orthogonal frequency-division multiplexing (OFDM) system using antennas with reconfigurable radiation patterns. In this system, each element of the antenna array at the transmitter side is assumed to be reconfigurable so that it can independently change the physical characteristics of its radiation pattern. The proposed block code is full rate and benefits from spatial, frequency, and reconfigurable radiation pattern state diversity over frequency-selective fading channels. We provide simulation results to demonstrate the performance of the proposed block coding technique and make comparisons with that of the previous SF coding schemes in MIMO-OFDM systems. The results indicate that the proposed code achieves higher diversity and coding gain compared to other available SF codes.

Index Terms-Frequency-selective fading channels, multipleinput multiple-output-orthogonal frequency-division multiplexing (MIMO-OFDM) systems, space-frequency (SF) coding, reconfigurable antennas.
\end{abstract}

\section{INTRODUCTION}

Reconfigurable antennas can be used in multiple-input multiple-output (MIMO) communication systems to increase the capacity and reliability of wireless links [1]-[5]. In a reconfigurable MIMO system, the characteristics of each antenna radiation pattern can be changed by placing switching devices such as Microelectromechanical Systems (MEMS), varactor diodes, or field-effect transistor (FET) within the antenna structure [6]-[8]. As a result, a system employing reconfigurable antennas is able to alter the propagation characteristics of the wireless channel into a form that leads to a better signal quality at the receiver. In fact, by using reconfigurable antennas and designing a proper code, we can achieve an additional diversity gain that can further improve the performance of wireless communication systems.

There are several works in the literature on designing efficient codes for reconfigurable MIMO systems in order to take advantage of the antenna reconfigurability. In [5], authors have proposed a MIMO system equipped with reconfigurable antennas at the receiver that can achieve a diversity order that equals to the product of the number of transmit antennas, the number of receive antennas and the number of reconfigurable states of the receive antennas. They have shown that this diversity gain is achievable only under certain channel propagation conditions and using an appropriate coding technique. Later on, in [9] authors extended the concept by using reconfigurable elements at both transmitter and receiver sides. In their work, they have introduced a state-switching transmission scheme, called space-time-state block coding (STS-BC), to further utilize the available diversity in the system over flat fading wireless channels. However, their coding scheme does not exploit the frequency diversity offered by the multipath propagation channels between each transmit and receive antenna pair.

To obtain frequency diversity in multipath environment, a space-frequency (SF) block code was first proposed by authors in [10], where they used the existing space-time (ST) coding concept and constructed the code in frequency domain. Later works [11]-[16] also used similar strategies to develop SF codes for MIMO-OFDM systems. However, the resulting SF codes achieved only spatial diversity, and they were not able to obtain both spatial and frequency diversities. To address this problem, a subcarrier grouping method has been proposed in [17] to further enhance the diversity gain while reducing the receiver complexity. In [18], a repetition mapping technique has been proposed that obtains full-diversity in frequencyselective fading channels. Although their proposed technique achieves full-diversity order, it does not guarantee full coding rate. Subsequently, a block coding technique that offers fulldiversity and full coding rate was derived [19], [20]. However, the SF codes proposed in the above studies and other similar works on the topic are not able to exploit the state diversity available in reconfigurable multiple antenna systems.

In this paper, we propose a coding scheme for reconfigurable MIMO-OFDM systems that achieves multiple diversity gains, including, space, frequency, and state. Basically, the proposed scheme consists of a code that is sent over transmit antennas, OFDM tones, and radiation states. In order to obtain state diversity, we configure each transmit antenna element to independently switch its radiation pattern to a direction that can be selected according to different optimization criteria, e.g., to minimize the correlation among different radiation 
states. We construct our proposed code based on the fundamental concept of rotated quasi-orthogonal space-time block codes (QOSTBC) [21]-[23]. By using the rotated QOSTBC, the proposed coding structure provides rate-one transmission (i.e., one symbol per frequency subcarrier per radiation state) and leads to a simpler Maximum Likelihood (ML) decoder. As the simulation results indicate, our proposed code outperforms the existing space-frequency codes substantially.

The rest of this paper is organized as follows. In Section we introduce the channel and system model for a reconfigurable MIMO-OFDM system. In Section $\Pi$ we briefly discuss the code design for reconfigurable multiple antenna systems. Simulation results are presented in Section IV and finally conclusions are drawn in Section $\mathrm{V}$

Notation: Throughout this paper, we use capital boldface letters for matrices, and lowercase boldface letters for vectors. $(\cdot)^{T}$ denotes transpose of a vector. $\mathcal{C}$ stands for the set of complex valued numbers. Operator $\operatorname{diag}\left(a_{1}, a_{2}, \cdots, a_{n}\right)$ represents a diagonal $n \times n$ matrix whose diagonal entries are $a_{1}, a_{2}, \cdots, a_{n} .\lfloor\cdot\rfloor$ stands for the floor operation and $\mathbf{I}_{N}$ represents the $N \times N$ identity matrix. Operator $\operatorname{col}\{\cdot\}$ stacks up the matrices on top of each other.

\section{Channel AND System Models FOR RECONFIGURABLE MIMO-OFDM SYSTEMS}

Consider a MIMO-OFDM system with $M_{t}$ reconfigurable elements at the transmitter where each of these elements is capable of electronically changing its radiation pattern and creating $P$ different radiation states as shown in Fig. 1 In this system, we assume the receiver antenna array consist of $M_{r}$ omni-directional elements with fixed radiation patterns. Moreover, we consider an $N_{c}$-tone OFDM modulation and frequency-selective fading channels with $L$ independent propagation paths between each pair of transmit and receiver antenna in each radiation state. The channel gains are quasistatic over one OFDM symbol interval. The channel impulse response between transmit antenna $i$ and receive antenna $j$ in the $p$-th radiation state can be modelled as

$$
h_{p}^{i, j}(\tau)=\sum_{l=0}^{L-1} \alpha_{p}^{i, j}(l) \delta\left(\tau-\tau_{l, p}\right),
$$

where $\tau_{l, p}$ is the $l$-th path delay in the $p$-th radiation state, and $\alpha_{p}^{i, j}(l)$ is the complex amplitude of the $l$-th path between the $i$-th reconfigurable transmit antenna and the $j$-th receive antenna in the $p$-th radiation state. The average total received power is normalized to one.

The frequency response of the channel at the $n$-th subcarrier between transmit antenna $i$ and receive antenna $j$ in the $p$-th radiation state is given by

$$
H_{p}^{i, j}(n)=\sum_{l=0}^{L-1} \alpha_{p}^{i, j}(l) e^{-j 2 \pi n \Delta f \tau_{l, p}},
$$

where $\Delta f=1 / T_{s}$ is the subcarrier frequency spacing and $T_{s}$ is the OFDM symbol duration. The space-frequency codeword transmitted during the $p$-th radiation state, $\mathbf{C}_{p} \in \mathcal{C}^{M_{t} \times N_{c}}$, can be expressed as

$$
\mathbf{C}_{p}=\left[\begin{array}{cccc}
c_{p}^{1}(0) & c_{p}^{1}(1) & \cdots & c_{p}^{1}\left(N_{c}-1\right) \\
c_{p}^{2}(0) & c_{p}^{2}(1) & \cdots & c_{p}^{2}\left(N_{c}-1\right) \\
\vdots & \vdots & \ddots & \vdots \\
c_{p}^{M_{t}}(0) & c_{p}^{M_{t}}(1) & \cdots & c_{p}^{M_{t}}\left(N_{c}-1\right)
\end{array}\right]
$$

where $c_{p}^{i}(n)$ denotes the data symbol transmitted by transmit antenna $i$ on the $n$-th subcarrier during the $p$-th radiation state.

At the receiver, after cyclic prefix removal and FFT, the received frequency domain signal of the $n$-th subcarrier and $p$-th radiation state at the $j$-th receive antenna can be written as

$$
y_{p}^{j}(n)=\sqrt{\frac{\gamma}{M_{t}}} \sum_{i=1}^{M_{t}} H_{p}^{i, j}(n) c_{p}^{i}(n)+z_{p}^{j}(n),
$$

where $H_{p}^{i, j}(n)$ is the frequency response of the channel at the $n$-th subcarrier between transmit antenna $i$ and receive antenna $j$ in the $p$-th radiation state as defined in (2), $z_{p}^{j}(n)$ is the additive complex Gaussian noise with zero mean and unit variance at the $n$-th subcarrier, and $\gamma$ is the received signal-to noise ratio (SNR).

The received signal during the $p$-th radiation state $\mathbf{y}_{p}=\left[\begin{array}{llll}\mathbf{y}_{p}^{T}(0) & \mathbf{y}_{p}^{T}(1) & \cdots & \mathbf{y}_{p}^{T}\left(N_{c}-1\right)\end{array}\right]^{T}$ with $\mathbf{y}_{p}(n)=$ $\left[y_{p}^{1}(n) y_{p}^{2}(n) \cdots y_{p}^{M_{r}}(n)\right]^{T}$, can be written as

$$
\mathbf{y}_{p}=\sqrt{\frac{\gamma}{M_{t}}} \mathbf{H}_{p} \mathbf{c}_{p}+\mathbf{z}_{p}
$$

where

$$
\mathbf{H}_{p}=\operatorname{diag}\left\{\mathbf{H}_{p}(0), \mathbf{H}_{p}(1), \cdots, \mathbf{H}_{p}\left(N_{c}-1\right)\right\}
$$

is the channel matrix, $\mathbf{c}_{p}=\operatorname{vec}\left(\mathbf{C}_{p}\right)$ is the transmitted codeword, and $\mathbf{z}_{p} \in \mathcal{C}^{N_{c} M_{r} \times 1}$ is the noise vector during the $p$-th radiation state. In (6), $\mathbf{H}_{p}(n)$ is an $M_{r} \times M_{t}$ channel matrix with entries defined in (2).

The SF codeword over all $P$ radiation states can be represented as

$$
\mathbf{C}=\left[\mathbf{C}_{1}, \mathbf{C}_{2}, \cdots, \mathbf{C}_{P}\right],
$$

where $\mathbf{C}_{p}$ is given in (3). The received signals over all radiation states is defined by $\mathbf{y}=\left[\begin{array}{llll}\mathbf{y}_{1}^{T} & \mathbf{y}_{2}^{T} & \cdots & \mathbf{y}_{P}^{T}\end{array}\right]^{T} \in \mathcal{C}^{P N_{c} M_{r} \times 1}$ and can be represented by

$$
\mathbf{y}=\sqrt{\frac{\gamma}{M_{t}}} \mathbf{H c}+\mathbf{z},
$$

where $\mathbf{c}=\operatorname{vec}(\mathbf{C}), \mathbf{H}=\operatorname{diag}\left\{\mathbf{H}_{1}, \mathbf{H}_{2}, \cdots, \mathbf{H}_{P}\right\} \in$ $\mathcal{C}^{P N_{c} M_{r} \times P N_{c} M_{t}}$ is the overall channel matrix, and $\mathbf{z}=$ $\left[\begin{array}{llll}\mathbf{z}_{1}^{T} & \mathbf{z}_{2}^{T} & \cdots & \mathbf{z}_{P}^{T}\end{array}\right]^{T} \in \mathcal{C}^{P N_{c} M_{r} \times 1}$ is the noise vector. 


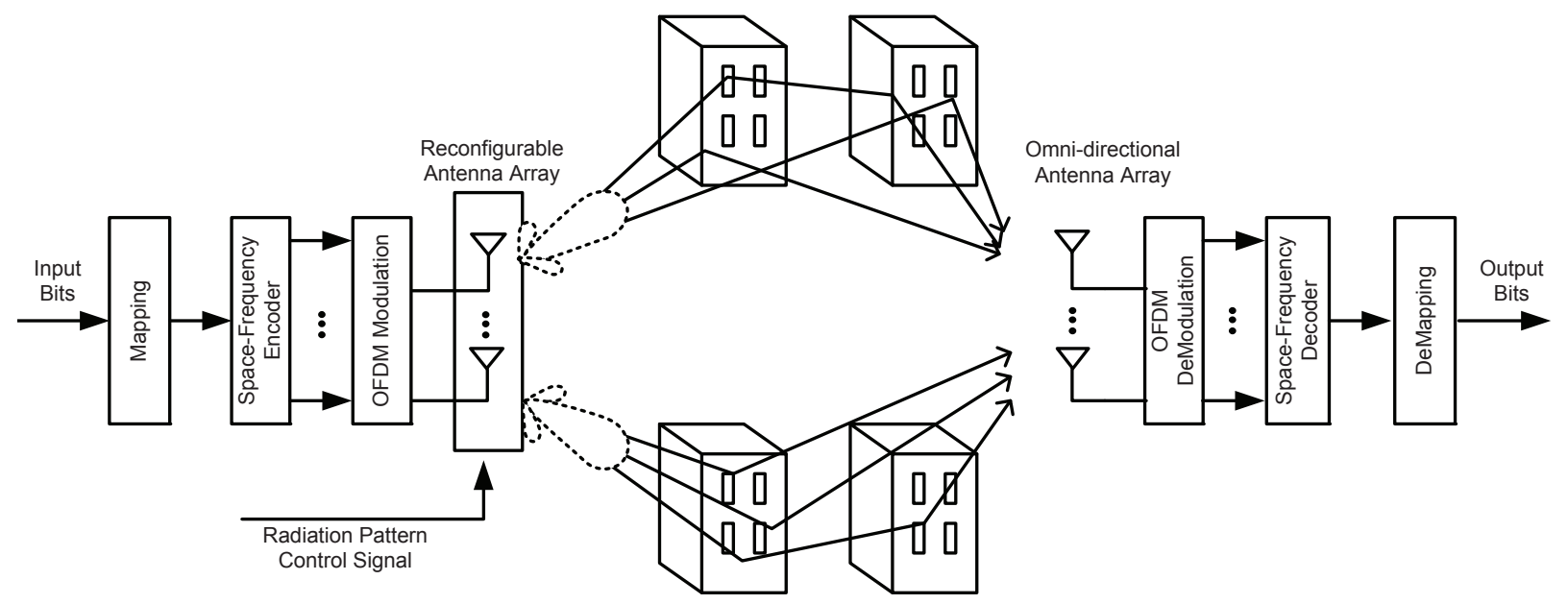

Fig. 1. Block diagram of a Reconfigurable MIMO-OFDM system employing reconfigurable antennas at the transmitter.

\section{Space-Frequency Code Design for RECONFIGURABLE MIMO-OFDM SYSTEMS}

In this section, we present our proposed coding scheme for a reconfigurable antenna system, where each antenna elements can independently change its radiation pattern direction. In particular, we construct the code based on the principle of a quasi-orthogonal coding structure for an arbitrary number of transmit antennas and radiation pattern states. In each radiation state, we consider a coding strategy where the SF codeword is a concatenation of $\mathbf{G}_{p}^{m^{T}}$ as follows:

$$
\mathbf{C}_{p}=\left[\mathbf{G}_{p}^{1^{T}} \mathbf{G}_{p}^{2^{T}} \cdots \mathbf{G}_{p}^{M^{T}} \mathbf{0}_{N_{c}-M L M_{t}}^{T}\right]
$$

where $M=\left\lfloor\frac{N_{c}}{L M_{t}}\right\rfloor$ and $\mathbf{0}_{N}$ is the all-zeros $N \times N$ matrix. In this expression, $\mathbf{0}_{N}$ will disappear if $N_{c}$ is an integer multiple of $L M_{t}$. In this work, for simplicity, we assume $N_{c}=L M_{t} q$, for some integer $q$. Each $\mathbf{G}_{p}^{m}$ matrix, $m \in\{1,2, \cdots, M\}$, takes the following form:

$$
\mathbf{G}_{p}^{m}=\operatorname{col}\left\{\mathbf{X}_{1}, \mathbf{X}_{2}, \cdots, \mathbf{X}_{L}\right\}
$$

where $\mathbf{X}_{l}$ is the $M_{t} \times M_{t}$ block coding matrix which is equivalent to an Alamouti code structure for $M_{t}=2$. To maintain simplicity in our presentation, we design the code for $M_{t}=2$ transmit antennas, however, extension to $M_{t}>2$ is possible by following the similar procedure. In the case of having two transmit antennas, $\mathbf{X}_{l}=\mathbf{A}\left(x_{1}, x_{2}\right)$, where

$$
\mathbf{A}\left(x_{1}, x_{2}\right)=\left[\begin{array}{cc}
x_{1} & x_{2} \\
-x_{2}^{*} & x_{1}^{*}
\end{array}\right],
$$

and therefore $\mathbf{G}_{p}^{m}$ can be expressed as

$$
\mathbf{G}_{p}^{m}=\left[\begin{array}{c}
\mathbf{A}\left(\mathcal{S}_{2(p-1) L+1}^{m}, \mathcal{S}_{2(p-1) L+2}^{m}\right) \\
\mathbf{A}\left(\mathcal{S}_{2(p-1) L+3}^{m}, \mathcal{S}_{2(p-1) L+4}^{m}\right) \\
\vdots \\
\mathbf{A}\left(\mathcal{S}_{2 p L-1}^{m}, \mathcal{S}_{2 p L}^{m}\right)
\end{array}\right] .
$$

In (12), $\mathcal{S}_{i}^{m}$ is a set of combined symbols, computed as

$$
\begin{aligned}
& {\left[\begin{array}{llll}
\mathcal{S}_{1}^{m} \mathcal{S}_{3}^{m} & \cdots & \mathcal{S}_{2 P L-1}^{m}
\end{array}\right]^{T}=\Theta\left[\begin{array}{llll}
s_{1}^{m} & s_{3}^{m} & \cdots & s_{2 P L-1}^{m}
\end{array}\right]^{T},} \\
& {\left[\begin{array}{lllll}
\mathcal{S}_{2}^{m} & \mathcal{S}_{4}^{m} & \cdots & \mathcal{S}_{2 P L}^{m}
\end{array}\right]^{T}=\Theta\left[\begin{array}{lllll}
s_{2}^{m} & s_{4}^{m} & \cdots & s_{2 P L}^{m}
\end{array}\right]^{T},}
\end{aligned}
$$

where $\left\{s_{1}^{m}, \cdots, s_{2 P L}^{m}\right\}$ is a block of symbols belonging to a constellation $\mathcal{A}, \Theta=\mathbf{U} \times \operatorname{diag}\left\{1, e^{j \theta_{1}}, \ldots, e^{j \theta_{P L-1}}\right\}$ and $\mathbf{U}$ is a $P L \times P L$ Hadamard matrix. The $\theta_{i}$ 's are the rotation angles. Different optimization strategies can be used to find the optimal values of rotation angles $\theta_{i}$ 's, such that they maximize the coding gain. The objective function in this optimization is defined as the minimum Euclidean distance between constellation points.

As an example, consider a reconfigurable MIMO-OFDM system with $M_{t}=2$ transmit antennas, $P=2$ radiation states, and $L=2$ multipaths. In this scenario, the transmitted codewords $\mathbf{C}_{1}$ and $\mathbf{C}_{2}$ given in (14) are constructed according

$$
\begin{aligned}
\mathbf{C}_{1} & =\frac{1}{4}\left[\begin{array}{ccccccccc}
\mathcal{S}_{1}^{1} & -\mathcal{S}_{2}^{1^{*}} & \mathcal{S}_{3}^{1} & -\mathcal{S}_{4}^{1^{*}} & \ldots & \mathcal{S}_{1}^{M} & -\mathcal{S}_{2}^{M^{*}} & \mathcal{S}_{3}^{M} & -\mathcal{S}_{4}^{M^{*}} \\
\mathcal{S}_{2}^{1} & \mathcal{S}_{1}^{1 *} & \mathcal{S}_{4}^{1} & \mathcal{S}_{3}^{1 *} & \cdots & \mathcal{S}_{2}^{M} & \mathcal{S}_{1}^{M *} & \mathcal{S}_{4}^{M} & \mathcal{S}_{3}^{M *}
\end{array}\right] \\
\mathbf{C}_{2} & =\frac{1}{4}\left[\begin{array}{ccccccccc}
\mathcal{S}_{5}^{1} & -\mathcal{S}_{6}^{1^{*}} & \mathcal{S}_{7}^{1} & -\mathcal{S}_{8}^{1^{*}} & \ldots & \mathcal{S}_{5}^{M} & -\mathcal{S}_{6}^{M^{*}} & \mathcal{S}_{7}^{M} & -\mathcal{S}_{8}^{M^{*}} \\
\mathcal{S}_{6}^{1} & \mathcal{S}_{5}^{1 *} & \mathcal{S}_{8}^{1} & \mathcal{S}_{7}^{1 *} & \cdots & \mathcal{S}_{6}^{M} & \mathcal{S}_{5}^{M *} & \mathcal{S}_{8}^{M} & \mathcal{S}_{7}^{M *}
\end{array}\right]
\end{aligned}
$$


to (9). The entries of $\mathbf{C}_{p}$ are computed using (13). As a result, we obtain $\mathbf{C}_{1}^{T}$ as

$$
\left[\begin{array}{cc}
s_{1}^{1}+\tilde{s}_{3}^{1}+\hat{s}_{5}^{1}+\check{s}_{7}^{1} & s_{2}^{1}+\tilde{s}_{4}^{1}+\hat{s}_{6}^{1}+\check{s}_{8}^{1} \\
-s_{2}^{1^{*}}-\tilde{s}_{4}^{1^{*}}-\hat{s}_{6}^{1^{*}}-\check{s}_{8}^{1^{*}} & s_{1}^{1^{*}}+\tilde{s}_{3}^{1^{*}}+\hat{s}_{5}^{1^{*}}+\check{s}_{7}^{*^{*}} \\
s_{1}^{1}-\tilde{s}_{3}^{1}+\hat{s}_{5}^{1}-\check{s}_{7}^{1} & s_{2}^{1}-\tilde{s}_{4}^{1}+\hat{s}_{6}^{1}-\check{s}_{8}^{1} \\
-s_{2}^{1^{*}}+\tilde{s}_{4}^{1^{*}}-\hat{s}_{6}^{1^{*}}+\check{s}_{8}^{1^{*}} & s_{1}^{1^{*}}-\tilde{s}_{3}^{1^{*}}+\hat{s}_{5}^{1^{*}}-\check{s}_{7}^{*^{*}} \\
\vdots & \vdots \\
s_{1}^{M}+\tilde{s}_{3}^{M}+\hat{s}_{5}^{M}+\check{s}_{7}^{M} & s_{2}^{M}+\tilde{s}_{4}^{M}+\hat{s}_{6}^{M}+\check{s}_{8}^{M} \\
-s_{2}^{M^{*}}-\tilde{s}_{4}^{M^{*}}-\hat{s}_{6}^{M^{*}}-\check{s}_{8}^{M^{*}} & s_{1}^{M^{*}}+\tilde{s}_{3}^{M^{*}}+\hat{s}_{5}^{M^{*}}+\check{s}_{7}^{M^{*}} \\
s_{1}^{M}-\tilde{s}_{3}^{M}+\hat{s}_{5}^{M}-\check{s}_{7}^{M} & s_{2}^{M}-\tilde{s}_{4}^{M}+\hat{s}_{6}^{M}-\check{s}_{8}^{M} \\
-s_{2}^{M^{*}}+\tilde{s}_{4}^{M^{*}}-\hat{s}_{6}^{M^{*}}+\check{s}_{8}^{M^{*}} & s_{1}^{M^{*}}-\tilde{s}_{3}^{M^{*}}+\hat{s}_{5}^{M^{*}}-\check{s}_{7}^{M^{*}}
\end{array}\right],
$$

and $\mathbf{C}_{2}^{T}$ as

$$
\left[\begin{array}{cc}
s_{1}^{1}+\tilde{s}_{3}^{1}-\hat{s}_{5}^{1}-\check{s}_{7}^{1} & s_{2}^{1}+\tilde{s}_{4}^{1}-\hat{s}_{6}^{1}-\check{s}_{8}^{1} \\
-s_{2}^{1^{*}}-\tilde{s}_{4}^{1^{*}}+\hat{s}_{6}^{1^{*}}+\check{s}_{8}^{1^{*}} & s_{1}^{1^{*}}+\tilde{s}_{3}^{1^{*}}-\hat{s}_{5}^{1^{*}}-\check{s}_{7}^{{ }^{*}} \\
s_{1}^{1}-\tilde{s}_{3}^{1}-\hat{s}_{5}^{1}+\check{s}_{7}^{1} & s_{2}^{1}-\tilde{s}_{4}^{1}-\hat{s}_{6}^{1}+\check{s}_{8}^{1} \\
-s_{2}^{1^{*}}+\tilde{s}_{4}^{1^{*}}+\hat{s}_{6}^{1^{*}}-\check{s}_{8}^{1^{*}} & s_{1}^{1^{*}}-\tilde{s}_{3}^{1^{*}}-\hat{s}_{5}^{1^{*}}+\check{s}_{7}^{1^{*}} \\
\vdots & \vdots \\
s_{1}^{M}+\tilde{s}_{3}^{M}-\hat{s}_{5}^{M}-\check{s}_{7}^{M} & s_{2}^{M}+\tilde{s}_{4}^{M}-\hat{s}_{6}^{M}-\check{s}_{8}^{M} \\
-s_{2}^{M^{*}}-\tilde{s}_{4}^{M^{*}}+\hat{s}_{6}^{M^{*}}+\check{s}_{8}^{M^{*}} & s_{1}^{M^{*}}+\tilde{s}_{3}^{M^{*}}-\hat{s}_{5}^{M^{*}}-\check{s}_{7}^{M^{*}} \\
s_{1}^{M}-\tilde{s}_{3}^{M}-\hat{s}_{5}^{M}+\check{s}_{7}^{M} & s_{2}^{M}-\tilde{s}_{4}^{M}-\hat{s}_{6}^{M}+\check{s}_{8}^{M} \\
-s_{2}^{M^{*}}+\tilde{s}_{4}^{M^{*}}+\hat{s}_{6}^{M^{*}}-\check{s}_{8}^{M^{*}} & s_{1}^{M^{*}}-\tilde{s}_{3}^{M^{*}}-\hat{s}_{5}^{M^{*}}+\check{s}_{7}^{M^{*}}
\end{array}\right]_{(16)}
$$

where $\tilde{s}_{i}=e^{j \theta_{1}} s_{i}, \hat{s}_{i}=e^{j \theta_{2}} s_{i}$, and $\check{s}_{i}=e^{j \theta_{3}} s_{i}$. Note that the above codeword provides rate-one transmission (i.e., one symbol per OFDM tone per radiation state).

\section{Simulation Results}

In this section, we present simulation results for both conventional and reconfigurable MIMO-OFDM systems. The reconfigurable multiple antenna system employs antenna elements capable of dynamically changing their radiation pattern directions at the transmitter, where in this work, we consider that each element has $P=2$ radiation states. The conventional MIMO-OFDM system uses omni-directional antenna elements with fixed radiation pattern at both transmitter and receiver ends. For both systems, we consider $M_{t}=2$ antennas at the transmitter and $M_{r}=1$ antenna at the receiver and an OFDM modulation technique with $N_{c}=128$ subcarriers as well as a cyclic prefix equal to or longer than the maximum channel delay spread. In our simulations, we consider that the receiver has perfect channel state information. We also assume that the symbols are chosen from a BPSK constellation, leading to a spectral efficiency of $1 \mathrm{bit} / \mathrm{sec} / \mathrm{Hz}$ if the cyclic prefix overhead is ignored. The average symbol power per transmit antenna is set to be $E_{s}=1 / M_{t}$ and the noise variance is $\sigma_{n}^{2}=1 / \gamma$. We carry out the simulations for a 2-ray equal power channel model for two different delay spreads. Furthermore, for a reconfigurable antenna system, we assume the same delay spread for both radiation states

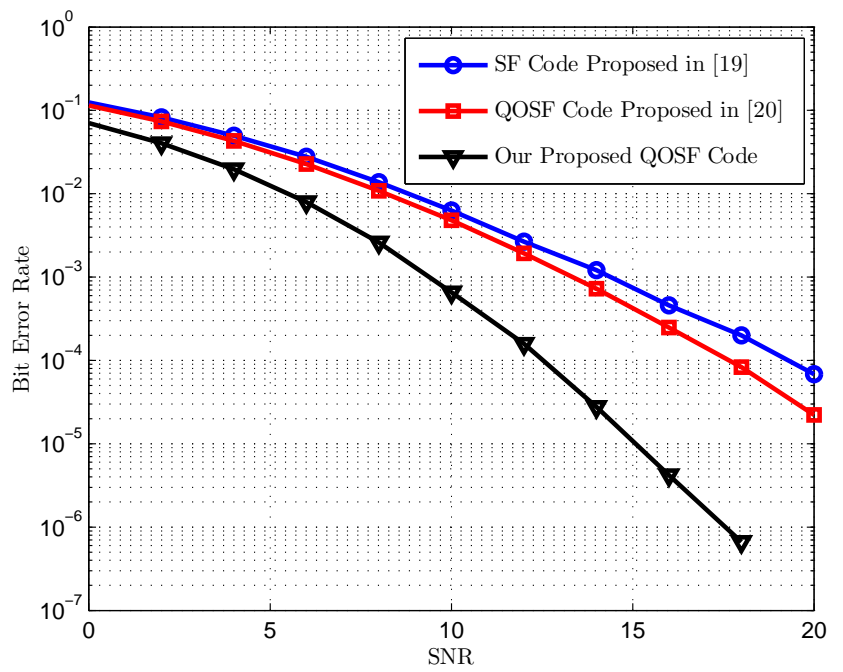

Fig. 2. BER vs. SNR for a reconfigurable multi-antenna system with $M_{t}=2$, $P=2, M_{r}=1$ in a 2-ray channel with a delay spread of $5 \mu s$

(i.e., $\left.\tau_{l, 1}=\tau_{l, 2}\right)$. The channel coefficients $\alpha_{p}^{i, j}(l)$ are zeromean identically-distributed Gaussian random variables with a variance of $\sigma_{l, p}^{2}$. We assumed that they are independent for each multipath, transmit antenna and radiation pattern state. The powers of all paths in each radiation state are normalized such that $\sum_{l=0}^{L-1} \sigma_{l, p}^{2}=1$. For our proposed QOSF scheme, the rotation angles are chosen as $\theta_{1}=\frac{\pi}{4}, \theta_{2}=\frac{\pi}{2}$, and $\theta_{3}=\frac{3 \pi}{4}$.

Fig. 2 shows the bit error rate (BER) performance of the proposed code in multipath propagation channels with a delay spread of $\tau=5 \mu \mathrm{s}$. As shown in this figure, the proposed code outperforms those of [19] and [20]. In particular, at a bit error rate of $10^{-5}$, the performance improvement compared to [19] and [20] is nearly 7 and $6 \mathrm{~dB}$, respectively. This performance improvement demonstrates the superiority of our proposed scheme which is due to the extra diversity gain offered by the use of reconfigurable antenna elements.

Fig. 3 depicts the BER performance of the proposed code for a delay spread of $\tau=20 \mu \mathrm{s}$. It is evident from the figure that at a BER of $10^{-5}$, our proposed coding scheme outperforms the codes presented in [19] and [20] by about 6 and $4 \mathrm{~dB}$, respectively. Compared to the results in Fig. 2] it can be seen that as delay spread increases, the BER performance improves. This is due to benefiting from lower correlation between subcarriers, and therefore higher frequency diversity in multipath propagation channels.

\section{CONCLUSions}

We proposed a space-frequency coding technique for MIMO-OFDM systems using antennas with reconfigurable radiation patterns. The proposed code is constructed based on the principle of quasi-orthogonal coding scheme and consists of a block of transmitted symbols expanding over space, frequency, and radiation state dimensions. We provided simulation results to demonstrate the performance of the proposed 


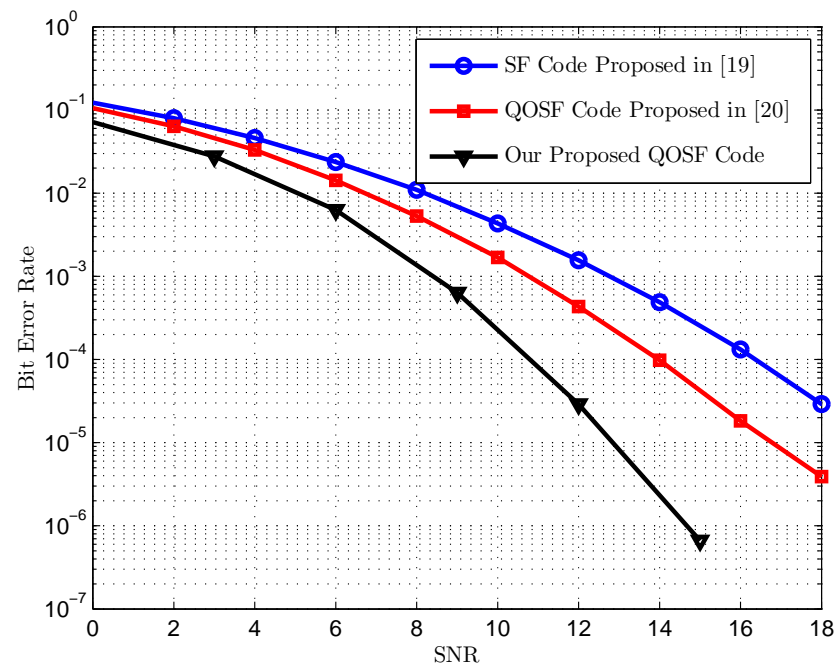

Fig. 3. BER vs. SNR for a reconfigurable multi-antenna system with $M_{t}=2$, $P=2, M_{r}=1$ in a 2-ray channel with a delay spread of $20 \mu \mathrm{s}$

coding scheme and make comparisons with that of the previous SF coding schemes. In these experiments, it has been shown that the proposed code provides additional diversity and coding gains compared to the previously designed SF codes in MIMO-OFDM systems.

\section{REFERENCES}

[1] B. Cetiner, E. Akay, E. Sengul, and E. Ayanoglu, "A MIMO system with multifunctional reconfigurable antennas," IEEE Antennas Wireless Propag. Lett., vol. 5, pp. 463-466, 2006.

[2] D. Piazza, N. Kirsch, A. Forenza, R. Heath, and K. Dandekar, "Design and evaluation of a reconfigurable antenna array for MIMO systems," IEEE Trans. Antennas Propagat., vol. 56, pp. 869-881, Mar. 2008.

[3] J. Frigon, C. Caloz, and Y. Zhao, "Dynamic radiation pattern diversity (DRPD) MIMO using CRLH leaky-wave antennas," in Proc. IEEE Radio and Wireless Symp., 2008, pp. 635-638.

[4] X. Li and J. Frigon, "Capacity analysis of MIMO systems with dynamic radiation pattern diversity," in Proc. IEEE VTC Spring 2009, 2009, pp. $1-5$.

[5] A. Grau, H. Jafarkhani, and F. De Flaviis, "A reconfigurable multipleinput multiple-output communication system," IEEE Trans. on Wireless Commun., vol. 7, pp. 1719-1733, May 2008.

[6] W. Weedon, W. Payne, and G. Rebeiz, "MEMS-switched reconfigurable antennas," in Antennas and Propagation Society International Symposium, 2001. IEEE, vol. 3, 2001, pp. 654-657.

[7] C. Caloz and T. Itoh, Electromagnetic metamaterials: transmission line theory and microwave applications. Wiley-IEEE Press, 2005.

[8] C. won Jung, M.-j. Lee, G. Li, and F. De Flaviis, "Reconfigurable scanbeam single-arm spiral antenna integrated with RF-MEMS switches," IEEE Trans. Antennas Propagat., vol. 54, pp. 455-463, Feb. 2006.

[9] F. Fazel, A. Grau, H. Jafarkhani, and F. Flaviis, "Space-time-state block coded MIMO communication systems using reconfigurable antennas," IEEE Trans. on Wireless Commun., vol. 8, pp. 6019-6029, Dec. 2009.

[10] D. Agrawal, V. Tarokh, A. Naguib, and N. Seshadri, "Space-time coded OFDM for high data-rate wireless communication over wideband channels," in Proc. IEEE Veh. Technol. Conf. (VTC), vol. 3, 1998, pp. 2232-2236.

[11] K. F. Lee and D. B. Williams, "A space-time coded transmitter diversity technique for frequency selective fading channels," in in Proc. IEEE Sensor Array and Multichannel Signal Processing Workshop, March 2000, pp. 149-152.

[12] H. Bolcskei and A. J. Paulraj, "Space-frequency coded broadband OFDM systems," in Proc. IEEE Wireless Commun. Networking Conf. (WCNC), vol. 1, Sept. 2000, pp. 1-6.
[13] B. Lu and X. Wang, "Space-time code design in OFDM systems," in Proc. IEEE Global Commun. Conf. (GLOBECOM), vol. 2, Nov. 2000 pp. 1000-1004.

[14] R. S. Blum, Y. G. Li, J. H. Winters, and Q. Yan, "Improved spacetime coding for MIMO-OFDM wireless communications," IEEE Trans. Commun., vol. 49, pp. 1873-1878, Nov. 2001

[15] Z. Hong and B. L. Hughes, "Robust space-time codes for broadband OFDM systems," in Proc. IEEE Wireless Commun. Networking Conf. (WCNC), vol. 1, 2002, pp. 105-108.

[16] K. F. Lee and D. B. Williams, "A space-frequency transmitter diversity technique for OFDM systems," in Proc. IEEE Global Commun. Conf. (GLOBECOM), vol. 3, 2000, pp. 1473-1477.

[17] A. F. Molisch, M. Z. Win, and J. H. Winters, "Space-time-frequency (STF) coding for MIMO-OFDM systems," IEEE Commun. Lett., vol. 6 , pp. 370-372, Sept. 2002.

[18] W. Su, Z. Safar, M. Olfat, and K. R. Liu, "Obtaining full-diversity spacefrequency codes from space-time codes via mapping," IEEE Trans Signal Process., vol. 51, no. 11, pp. 2905-2916, Nov. 2003.

[19] W. Su, Z. Safar, and K. R. Liu, "Full-rate full-diversity space-frequency codes with optimum coding advantage," IEEE Trans. Inf. Theory, vol. 51, pp. 229-249, Jan. 2005.

[20] F. Fazel and H. Jafarkhani, "Quasi-orthogonal space-frequency and space-time-frequency block codes for MIMO OFDM channels," IEEE Trans. on Wireless Commun., vol. 7, pp. 184-192, Jan. 2008.

[21] O. Tirkkonen, "Optimizing space-time block codes by constellation rotations," in Finnish Wireless Commun. Workshop (FWWC), Oct. 2001, pp. 59-60.

[22] N. Sharma and C. B. Papadias, "Improved quasi-orthogonal codes through constellation rotation," IEEE Trans. Commun., vol. 51, pp. 332335, March 2003.

[23] W. Su and X.-G. Xia, "Signal constellations for quasi-orthogonal spacetime block codes with full diversity," IEEE Trans. Inf. Theory, vol. 50, pp. 2331-2347, Oct. 2004 